December 1, 1999 - Astrophysical Journal, in press

\title{
Observations and Models of a Flaring Loop
}

\author{
A. Nindos, S. M. White, M. R. Kundu \\ Astronomy Department, University of Maryland, College Park, MD 20742 \\ D. E. Gary \\ Physics Department, NJIT, Newark, NJ 07102
}

\begin{abstract}
Simultaneous images of a flaring loop at two frequencies are used to model the magnetic structure of the loop and the energy distribution of the radiating electrons. The imaging data were obtained with the VLA at 5 and $15 \mathrm{GHz}$. Additional spectral data were provided by the OVRO Solar Array at several frequencies between $2 \mathrm{GHz}$ and $15 \mathrm{GHz}$. At $15 \mathrm{GHz}$, the flare emission was optically thin and came from the footpoints of the flaring loop, while at $5 \mathrm{GHz}$ the loop itself was outlined. Most of the $5 \mathrm{GHz}$ emission was optically thick and its spatial maximum was close to the loop top. A striking feature of the observations is that the $5 \mathrm{GHz}$ emission does not reach down to the $15 \mathrm{GHz}$ footpoints. We compare the observations with calculations of gyrosynchrotron emission from an inhomogeneous magnetic loop in order to determine the conditions in the flaring loop. The best fit to the OVRO fluxes was reached with a model flaring loop with photospheric footpoint magnetic field strength of $870 \mathrm{G}$. The thickness of the model loop was small compared to its footpoint separation. The energy spectral index of the energetic electrons was 3.7 and their number density was $7.9 \times 10^{7} \mathrm{~cm}^{-3}$. The low and high energy cutoffs of the nonthermal electrons were 8 and $210 \mathrm{keV}$. The $5 \mathrm{GHz}$ emission in this model is at low harmonics $(3-7)$ and harmonic effects are responsible for the weak $5 \mathrm{GHz}$ emission at the footpoints. The absence of electrons above $210 \mathrm{keV}$ is necessary in this model to explain why no emission is observed from the loop top at $15 \mathrm{GHz}$. That model reproduced well the high frequency part of the OVRO flux spectrum as well as the VLA spatial structure. Thus comparisons between the spatially-resolved observations and models reveal the three-dimensional structure of the loop geometry.
\end{abstract}

Subject headings: Sun: flares - Sun: corona - Sun: radio radiation 


\section{Introduction}

Observations of solar microwave bursts can provide important diagnostics of acceleration processes in the solar corona because the radio emission is produced by energetic electrons accelerated during the flare. It is well known (e.g. see the reviews by Kundu, \& Vlahos 1982, Alissandrakis 1986; Bastian, Benz, \& Gary 1998) that the basic emission mechanism of solar microwave bursts is gyrosynchrotron from mildly relativistic electrons (energies of tens to several hundreds of keV) trapped in flaring loops. Gyrosynchrotron emission offers a powerful diagnostic of physical conditions in flaring regions. Unlike X-ray radiation, it is sensitive to magnetic field strength and orientation and can therefore be used to constrain the coronal magnetic field in the flaring source.

To a first approximation, the morphology of microwave flare emission depends on the magnetic field configuration (i.e. the geometry of the flaring region and its location on the disk) and the properties of the nonthermal electrons. Simultaneous microwave, soft X-ray and/or $\mathrm{H} \alpha$ observations of flares show that the magnetic configuration can be arcades of loops (e.g. Bastian, \& Kiplinger 1991), interacting loops of different size (Nishio et al. 1997; Hanaoka 1997) or more complex loop systems (e.g. Kundu et al. 1982; Velusamy, \& Kundu 1982; Takakura et al. 1994). Therefore, it is not surpising that high spatial resolution microwave observations of bursts have revealed a variety of morphologies. The reader may refer to the three extensive review papers cited above for a detailed description of previous observations. However, we note that the general tendency is that observations at high frequencies show compact sources, presumably associated with the footpoints of flaring loops, while at low frequencies there is a tendency for more extended sources, indicating emission from the entire loop. This picture is consistent with the properties of gyrosynchrotron mechanism: the magnetic field is much stronger near the footpoints of the loop, which favors the higher frequencies. A decrease of the frequency of observation has approximately the same effect for the microwave flare emission as an increase of the magnetic field. Thus when the frequency of observation decreases, we expect to have emission not only from the footpoints but also from a significant part of the flaring loop.

Since source morphology may vary as a function of frequency, spatially resolved spectral observations are needed in order to obtain a complete picture of the radio flare. However, in most cases images are not available and spatially unresolved spectra are used for the determination of the energy spectral index of the nonthermal electrons (e.g. Stähli, Gary, \& Hurford 1989). Occasionally, imaging spectroscopy of simple events has been made with the OVRO solar array (Wang et al., 1994, 1995). These observations have shown that, due to the change in magnetic field strength in the loop, low frequency radio emission is concentrated near the top of the loop, and the centroid moves progressively down toward 
the footpoint at higher frequencies. For this reason, the looptop spectrum falls off more steeply at high frequencies than does the footpoint spectrum.

Inhomogeneous models of the microwave spatial structure of flaring loops have been computed by Alissandrakis, \& Preka-Papadema (1984), Preka-Papadema, \& Alissandrakis (1988, 1992) and Bastian, Benz, \& Gary (1998). All of them give a satisfactory qualitative interpretation of the elementary loop burst emission described above. Similar models of stellar loops have been calculated by Jones et al. (1994) and Franciosini \& Chiuderi-Drago (1995) . However, in most previous studies (the paper by Preka-Papadema et al. 1997 is probably the only exception) no attempt has been made at detailed modeling of the spatial structure of individual high resolution observations of bursts. In this paper, we study a simple small flare which was observed at $15 \mathrm{GHz}$ and $5 \mathrm{GHz}$ with the VLA. Additional total power flux spectra were obtained by OVRO. We try to reproduce the observed microwave morphology of the VLA maps as well as the OVRO flux spectrum using an inhomogeneous model of gyrosynchrotron emission. Comparison between the observations and models will

allow us to derive constraints about the magnetic configuration of the flaring region and the physical properties of the nonthermal electrons.

\section{Data Analysis}

The VLA observations were carried out on July 1, 1992. The CD configuration was used to image the area around the west sunspot of active region NOAA 7216 at $4.86 \mathrm{GHz}$ $(5 \mathrm{GHz})$ and $14.98 \mathrm{GHz}(15 \mathrm{GHz})$. The heliographic coordinates of the center of the field of view at both frequencies were N15E53. The observations lasted only 76 min (from 17:02 UT to 18:18 UT). The instrument was split into two subarrays, observing at 5 and $15 \mathrm{GHz}$, respectively. This yielded simultaneous coverage at two frequencies at the cost of reduced mapping ability. The flare studied here occurred from 17:17 UT to 17:40 UT. The VLA's high-time-resolution gating processor was used to observe with a time resolution of 0.4 sec but with the two circular polarizations gated so that each is integrated for alternate $0.2 \mathrm{sec}$ periods. However, an inspection of plots of the visibility amplitudes as a function of time showed no rapid fluctuations of the radio emission, and we therefore average the original $\mathrm{u}-\mathrm{v}$ databases so that the effective time resolution is $5 \mathrm{sec}$. The standard CLEAN algorithm was used to produce snapshot images of the radio emission during the time of the flare. We used self-calibration to improve the dynamic range of the snapshot maps. In order to maintain positional consistency, we used the CLEAN image from the preceding interval as the model at each step. For a slowly-varying source such as this, this procedure should work well. Inspection of burst movies made without the application of this technique 
showed that the radio sources danced around due to phase errors, particularly at $15 \mathrm{GHz}$ where the atmosphere has more effect on measured phases. The self-calibration procedure removed this motion. The final dynamic ranges of the snapshot maps were 25 and 17 for 5 $\mathrm{GHz}$ and $15 \mathrm{GHz}$ total intensity maps, respectively. The CLEAN beams of the final maps were about $7.9^{\prime \prime} \times 14^{\prime \prime}$ at $5 \mathrm{GHz}$ and $3.7^{\prime \prime} \times 3.1^{\prime \prime}$ at $15 \mathrm{GHz}$.

The spectral data consisted of total power fluxes obtained with the two 27-m OVRO antennas (antennas 1 and 2) at 45 frequencies between $18 \mathrm{GHz}$ and $1 \mathrm{GHz}$ at a time resolution of $12 \mathrm{sec}$. In our study, we did not use the fluxes at low frequencies (from 1 to 2 $\mathrm{GHz}$ ) because at those frequencies the data from antennas 1 and 2 were not consistent with each other.

A KPNO full-disk magnetogram was available. The flare occurred during the period when the magnetogram was obtained. Unfortunately, neither $\mathrm{H} \alpha$ nor Yohkoh observations were available during the flare. No BATSE hard X-ray data were available for this event.

\section{Radio Observations}

In Figure 1 we present contour plots of the VLA observations in total intensity, $I$, and circular polarization, $V$, at 5 and $15 \mathrm{GHz}$ at the time of maximum. The white contours represent the steady $15 \mathrm{GHz}$ sunspot-associated emission. No signature of that sunspot was found at $5 \mathrm{GHz}$ in either the flare snapshot maps or the map made with the non-flare data. We believe that the $5 \mathrm{GHz}$ active region emission was too complex to be mapped using only 53 min of observations obtained with the 13 antennas of the $5 \mathrm{GHz}$ subarray; it does not affect our flare images significantly because the likely peak brightness temperature of the active region emission, $3 \mathrm{MK}$, is much smaller than the typical brightness temperature of the flare at $5 \mathrm{GHz}, 20 \mathrm{MK}$. The lowest $5 \mathrm{GHz}$ contour of Fig. 1 is at about the level expected from the brightest active region emission.

At $15 \mathrm{GHz}$, the flare showed two compact sources presumably associated with the footpoints of a magnetic loop system filled with energetic electrons. The bulk of the loop is seen at $5 \mathrm{GHz}$. We note that a synthesis instrument is not sensitive to large sources due to the lack of sufficiently short baselines and for fixed antennas this problem is worse at higher frequencies. We must therefore address the question of whether the absence of emission from the looptop at $15 \mathrm{GHz}$ is a real effect or due to the lack of sensitivity of the $15 \mathrm{GHz}$ data to sources as large as the loop seen at $5 \mathrm{GHz}$. We therefore produced an artificial $15 \mathrm{GHz}$ observation of the loop by convolving the original $15 \mathrm{GHz} \mathrm{u}^{-\mathrm{v}}$ samples with a 5 $\mathrm{GHz}$ model image. The model image we used was the snapshot map which corresponded to 
the peak of the $5 \mathrm{GHz}$ event. The resulting data were mapped and cleaned as before. The resulting $15 \mathrm{GHz}$ map clearly shows the loop of the $5 \mathrm{GHz}$ model image, but with two small negative depressions being present as well. $70 \%$ of the original model flux was recovered in the $15 \mathrm{GHz}$ map. We conclude that the VLA $15 \mathrm{GHz}$ images would show a loop if there was $15 \mathrm{GHz}$ emission from a loop like that seen at $5 \mathrm{GHz}$; the absence of loop emission at $15 \mathrm{GHz}$ implies that any such emission must be much weaker than the footpoint emission. As we pointed out in section 1, the magnetic field is much stronger near the footpoints of the loop, which favors the higher frequency. The peak of the $5 \mathrm{GHz}$ emission is located close to the middle (or top) of the loop feature. Usually, loop top maxima occur when the emission is optically thick because the magnetic field is lowest at the loop top and therefore the effective energy of the electrons emitting there is higher. This was directly confirmed by the OVRO spectral data, which show that the emission peaked at $5.4 \mathrm{GHz}$ (see Fig. 3). Note a remarkable feature of the images: the $5 \mathrm{GHz}$ loop emission does not overlap with the peak $15 \mathrm{GHz}$ emission, particularly at the eastern footpoint. This implies that the bright $5 \mathrm{GHz}$ emission does not extend all the way down to the feet of the loop. This effect was sometimes more or less prominent than in Fig. 1 but it was present in almost all snapshot images that we produced. We confirmed the relative position of the 5 and $15 \mathrm{GHz}$ images by noting that the $5 \mathrm{GHz}$ snapshot images show no significant image motion from one snapshot to the next. We can therefore assume that the $5 \mathrm{GHz}$ position corresponds to the observed position, which is known to high precision relative to a nearby astrometric reference source. The $15 \mathrm{GHz}$ images can be located on the Sun thanks to the presence of the sunspot source whose boundaries match the umbra of the sunspot seen in the Kitt Peak magnetogram. We estimate that the uncertainty in the relative positions of the 5 and 15 $\mathrm{GHz}$ images is of order $1^{\prime \prime}$, which is much smaller than the offset of the $15 \mathrm{GHz}$ footpoint emission from the bottom of the $5 \mathrm{GHz}$ loop.

The $V$ maps of the flare show that both $15 \mathrm{GHz}$ footpoint sources were left-hand circularly polarized and the same was also true of the bulk of the $5 \mathrm{GHz}$ emission. The sense (but usually not the degree) of polarization can be affected if there is a neutral line in the corona along the line of sight: the effect is to reverse the sense of polarization of the limbward source. The sources on the near side of the neutral line in the active region generally show their intrinsic polarization. In this case, the polarizations of the emission of the sources on the near side of the neutral line (the sources to the west) are both consistent with extraordinary ( $x$ mode) mode polarization relative to the direction of the underlying magnetic field (negative polarity, i.e., downgoing). This identification is confirmed by the polarization of the $15 \mathrm{GHz}$ sunspot source, which should be $x$ mode and matches the polarization of the western $15 \mathrm{GHz}$ footpoint. The degrees of polarization of the $15 \mathrm{GHz}$ footpoint sources are typically $55 \%$ for the eastern footpoint and $25 \%$ for the western 
footpoint but reached values as high as $80-90 \%$ and $45-50 \%$ respectively. Such high degrees of polarization imply that the $15 \mathrm{GHz}$ emission was optically thin. At $5 \mathrm{GHz}$ the degree of polarization is typically $20 \%$ near the loop top, and decreases as one approaches the footpoints.

In fig. 2 we present the time profiles of the 5 and $15 \mathrm{GHz}$ VLA emission. The VLA fluxes were computed by summing up all pixels in the flare images with brightness temperatures higher than the lowest contours of fig. 1. The $5 \mathrm{GHz}$ profiles are simple; they show a rapid increase to the maximum intensity (within $2 \mathrm{~min}$ ) and a much slower decay. The $15 \mathrm{GHz}$ time profiles show that the microwave emission was higher at the eastern footpoint with the exception of the period 17:19:45 - 17:20:23 UT when the peak of the western footpoint emission was a factor of 3.6 higher than the eastern footpoint emission. This may indicate a secondary precipitation of energetic electrons above the western footpoint of the flaring loop. The morphology of the corresponding $5 \mathrm{GHz}$ maps did not change but a local peak in the $5 \mathrm{GHz} V$ time profile is clearly present about 5 sec before the peak of the $15 \mathrm{GHz}$ western footpoint emission.

Fig. 3 shows the OVRO total power fluxes from antennas 1 and 2 at the time of maximum of the event. The turnover frequency was $5.4 \mathrm{GHz}$. The OVRO and VLA fluxes at both 5 and $15 \mathrm{GHz}$ were consistent to within $20 \%$, which is acceptable given the uncertainties in instrumental calibration. We used the OVRO total power fluxes from 6 $\mathrm{GHz}$ to $15 \mathrm{GHz}$ to compute the slope $\alpha$ of the high frequency part of the flare spectrum. Once $\alpha$ is known, the computation of the electron energy spectral index $\delta$ is straightforward provided that the emission is optically thin $(\alpha=1.22-0.90 \delta$, Dulk 1985). We find that $\delta=3.7$. Due to local interference problems at the right circular feed of antenna 2, the low frequency part of the OVRO right-hand polarization (RCP) flux spectra (from $1 \mathrm{GHz}$ to $2 \mathrm{GHz}$ ) was not reliable; thus we have no information about the polarization of the flare at low frequencies. At high frequencies the OVRO fluxes show left circular polarization, in rough quantitative agreement with the fluxes from the VLA $V$ maps (differences about $20 \%$ ). The OVRO polarization spectra also show that from $2.8 \mathrm{GHz}$ to $4 \mathrm{GHz}$, there is a reversal of polarization.

\section{Model Computations}

\subsection{Input parameters}

The morphology of the radio images and the mostly slow nature of temporal variability clearly suggest that the radio emission arises from nonthemal electrons trapped in a loop. In 
this section we will use the data in conjunction with model calculations of gyrosynchrotron emission to determine the physical parameters of the loop. The parameters we investigate are as follows:

Magnetic loop geometry. The exact nature of the magnetic geometry cannot be determined since it can be quite complex. For modeling we require a geometry in which the field lines form natural loops, and choose for simplicity a line-dipole magnetic field. The field lines are circles with a common tangent point at the dipole: the magnetic field falls off quandratically with distance from the line dipole. We adopt the field of an infite line dipole truncated at a length corresponding to the length of the arcade of loops which we are modeling. The physical parameters of most interest are the magnetic field strengths at the footpoints and at the loop top. We will therefore not attempt to reproduce the spatial emission profiles in detail, but will focus on reproducing the behavior at the footpoints and at the loop top. In the line dipole configuration, the magnetic field strengths at loop top and footpoints are controlled by the height of the loop and the separation of the footpoints: a large ratio of height to footpoint separation yields a large ratio of footpoint field strength to loop-top field strength. There is no variation in the direction orthogonal to the plane of the loop (parallel to the line dipole) and we merely specify a loop thickness corresponding to the dimension of the source in this direction. The thickness is scaled along the loop by $B^{-0.5}$ in order to simulate magnetic flux conservation.

Loop orientation. In the simplest version of a line dipole the field lines lie in vertical planes above the dipole and orthogonal to the dipole axis. The heliographic latitude and longitude affect the appearance of the loop. In our code, the loop can be placed at any heliographic latitude and longitude with an arbitrary orientation with respect to the local north. We choose a set of parameters so that the model loop visually resembles the observed loop.

Energy spectrum. We assume that the energy distribution of the radiating electrons is a power-law over a finite energy range. Four parameters characterize the energy spectrum: the low energy cutoff, the high energy cutoff, the power-law slope and the total number of electrons in the distribution. We are assuming a trapped distribution isotropic in pitch angle. The assumption of a trapped distribution merely means that time variations should occur on a timescale much longer than the bounce period for an electron in the loop, which is less than a second for a $100 \mathrm{keV}$ electron in a loop $2 \times 10^{9} \mathrm{~cm}$ long.

Once these parameters are specified, we compute gyrosynchrotron emission from the 
loop using a code in which the gyrosynchrotron emissivity and opacity are calculated exactly at specified points along the loop, and the emitted radio flux is calculated using simple radiative transfer (see Schmahl, Kundu, \& Dennis 1986; Nitta et al. 1991). The extraordinary and ordinary mode emissions are calculated separately, and the standard quasi-longitudinal approximation to the electromagnetic mode properties is used (Zheleznyakov 1970).

The footpoint separation of the model loop is determined from the $15 \mathrm{GHz}$ VLA images: it is $d=2.6 \times 10^{9} \mathrm{~cm}$ which corresponds to the observed separation of the eastern and western $15 \mathrm{GHz}$ sources. The size $L$ of the $15 \mathrm{GHz}$ sources is about $5.3 \times 10^{8} \mathrm{~cm}$ and imposes an upper limit on the transverse dimension of the model loop (because our final maps are the convolution of the sky brightness distribution with the VLA beam). The radio spectral index from the OVRO data gives a power-law energy spectral index of $\delta=3.7$ (see section 3 ). The remaining parameters are the number density of the nonthermal electrons, their lower and upper cutoff energies, the heights above the solar surface of the lines of force of the magnetic field which form the loop and the strength of the magnetic field at the photosphere. (We do not have suitable magnetic field data for a nonlinear force-free reconstruction of the magnetic parameters from photospheric measurements.) The orientation of the loop is also variable subject to the constraint that it should resemble the observed loop. In particular, the model loop always lies in a vertical plane above the surface whereas the real loop may be tilted: we can simulate this by changing the latitude of the apparent loop.

Loughhead, Wang, \& Blows (1983) developed a geometrical method for the reconstructing the true shape of loops observed in solar images. Their formulas give the azimuth angle $\zeta$, defined as the angle between the line joining the footpoints and the local direction of solar west, and the inclination angle $\beta$ between the plane of the loop and the local vertical plane. They assume that the loop's central axis lies in a plane, the footpoints can be located and the loop is symmetrical about its top. Applying their formulas (10), (11), (16) and (17) to the $5 \mathrm{GHz}$ flare loop assuming the footpoints to be the $15 \mathrm{GHz}$ sources, we find $\zeta=24^{\circ}$ and $\beta=65^{\circ}$. However, these values are subject to uncertainty because the assumption that the loop is symmetrical about its top may not be correct. The value of $\beta$ that we find differs from recent results for other loops from SOHO EIT (e.g. Aschwanden et al. 1999) or older results from $\mathrm{H} \alpha$ data (Loughhead, Wang, \& Blows 1983) which give lower values of $\beta$. This discussion indicates that ultimately the loop orientation should be regarded as a free parameter in our program. 


\subsection{Results}

As a first attempt to model the observations we find a set of free parameters which reproduces the OVRO spectrum. We computed model total intensity fluxes at the same frequencies that OVRO data were available from $15 \mathrm{GHz}$ to $2 \mathrm{GHz}$, using the fixed physical dimensions described above and varying the remaining parameters. The best fit to the OVRO fluxes at the peak of the event was obtained with a model loop located at heliographic longitude $50^{\circ}$ east and latitude $50^{\circ}$ north and with a $45^{\circ}$ orientation (azimuth) with respect to the local north. The photospheric magnetic field stength at the footpoints of the model loop was $870 \mathrm{G}$. The heights above the solar surface of the upper and lower lines of force of the magnetic field which formed the loop were $1.9 \times 10^{9} \mathrm{~cm}$ and $1.8 \times 10^{9}$ $\mathrm{cm}$, respectively. The transverse dimension of the loop was $5.25 \times 10^{7} \mathrm{~cm}$. The loop was filled with energetic electrons with density $N_{e}=7.9 \times 10^{7} \mathrm{~cm}^{-3}$ and low and high energy cutoffs at $8 \mathrm{keV}$ and $210 \mathrm{keV}$, respectively. Fig. 3 shows the model spectrum (dotted line) and the OVRO spectra from antennas 1 and 2. The agreement between the model fluxes and the observations is excellent at high frequencies. The turnover frequency of the model flux spectrum was $5-5.2 \mathrm{GHz}$ while the observed turnover frequency was $5.4 \mathrm{GHz}$. However, the low frequency part of the spectrum was not reproduced as satisfactorily as the high frequency part: the discrepancies between the model and the observations were as high as a factor of 2.5. Steep low-frequency flux spectra have been observed several times with OVRO (e.g. see Stähli, Gary, \& Hurford 1989). The only way to match better the low frequency model fluxes to the corresponding OVRO fluxes was either to use a thinner loop or an electron distribution with a lower high-energy cutoff and/or a lower low-energy cutoff. But all cases produced much lower fluxes at high frequencies.

The Razin effect (Razin 1960; Klein 1987; Belkora 1997) is not included in our computations. The fact that the discrepancies generally increase as the frequency decreased may imply that indeed the disagreement was due to the Razin effect. However, this interpretation has problems because most of the model flux at low frequencies comes from regions close to the top of the loop which correspond to coronal heights with lower densities. We think it more likely that the discrepancies are due to the over-simplified loop model we use: this affects the optically-thick geometry-dependent low-frequency part of the spectrum more than the high-frequency optically-thin part. Thermal free-free absorption along the line of sight by overlying loops may provide an alternative explanation for the discrepancy. This process requires high-density, low-temperature thermal plasmas. Unfortunately, we cannot check this speculation due to the lack of suitable data.

We do not attempt to reproduce the OVRO circular polarization fluxes in detail with our model because our calculations do not include mode coupling effects outside the loop 
which will affect the sign of the polarized fluxes. We do however use the spatial distribution of the polarized flux in the following modifications of this fit to the spectrum.

In fig. 4 we present the morphologies at 4.86 and $14.98 \mathrm{GHz}$ of the model fit to the spectrum. In fig. 5 we show contour plots of the same models after we convolved each model with the appropriate VLA beam. In fig. 6 we compare a one-dimensional profile of the VLA data at the time of maximum flux with the model fluxes as a function of the distance along the loop, convolved with the appropriate VLA beam. The VLA profiles were computed along the curve shown in fig. 1. The model computations indicate that at $15 \mathrm{GHz}$ the radio emission is about $50 \%$ polarized and optically thin and comes from the footpoints of the loop. The $15 \mathrm{GHz}$ model footpoint emission is asymmetric and the highest emission was associated with the limbward footpoint of the loop. This is true even though the magnetic field strength in the footpoints of the model loop was symmetric. Alissandrakis \& Preka-Papadema (1984) and Preka-Papadema \& Alissandrakis (1988) have shown that for an east-west loop located away from the disk center, the peak of the microwave emission lies at the diskward part of the loop. However, this result is not appropriate for our observations and models because our loop is not oriented in the east-west direction. In our models, the strongest $15 \mathrm{GHz}$ source is associated with the limbward footpoint of the loop because the orientation of the model loop is such that the values of the angle between the magnetic field and the line of sight are higher in the limbward part of the loop. Preka-Papadema \& Alissandrakis (1992) have also shown that two-dimensional high-azimuth optically-thin model loops located away from the disk center may produce peak microwave emission close to their limbward parts in agreement with our computations.

At $5 \mathrm{GHz}$ the model emission comes from the entire loop. Most of the emission is optically thick with the exception of the east-central part of the loop where the optical depth is close to 1. Our model footpoint magnetic field is strong enough such that the entire $5 \mathrm{GHz}$ emission comes from low harmonics of the gyrofrequency (harmonics $3-7$ ) and the harmonic structure in the $I$ model emission is clearly seen in fig. 4. The peak of circular polarization corresponds to the area where the optical depth is close to unity. Local $V$ peaks are also present at the edges of the $I$ harmonic structures. The convolution of the models with the VLA beam gives a total intensity peak at the central-west part of the loop and a circular polarization peak at the central-east part of the loop (see fig. 5).

Overall, the model resulting from the fit to the spectrum reproduced most of the spatial features of the VLA observations in a qualitatively satisfactory way: both the model and the observations show optically thin footpoint emission at $15 \mathrm{GHz}$ and emission from the entire loop at $5 \mathrm{GHz}$. The model $15 \mathrm{GHz}$ emission was asymmetric with the strongest source located at the limbward part of the loop in agreement with the observations. The 
observation show that at $15 \mathrm{GHz}$, the eastern footpoint source was a factor of 1.7 stronger than the western footpoint source. In our model this ratio is 1.5 after the convolution. The model $5 \mathrm{GHz}$ emission peaks close to the loop top. But the peak is not as prominent as it is in the observations. Furthermore, the bulk of the $5 \mathrm{GHz} I$ model bright emission does not overlap with the $15 \mathrm{GHz}$ model sources in agreement with the observations. After convolution with the VLA beam, the $5 \mathrm{GHz} V$ profile shows a peak close to loop top but displaced with respect to the $I$ peak.

By changing the input parameters, we checked whether it is possible to achieve an even better agreement between the model and the observed morphologies at $5 \mathrm{GHz}$ and $15 \mathrm{GHz}$. Changes to the parameters have the following effects:

Magnetic field strength. A larger magnetic field strength increases the opacity and thus decreases the number density of electrons required to produce the same optically-thin flux. It also decreases the effective harmonic at which the electrons are radiating at a given frequency, which means that electrons are capable of producing optically thick $5 \mathrm{GHz}$ emission from the low-field central-east region at the loop top if, e.g., the footpoint field reaches $1000 \mathrm{G}$. An even larger field strength is required to give loop top emission at 15 $\mathrm{GHz}$. A field as small as $600 \mathrm{G}$ at the footpoints means that $15 \mathrm{GHz}$ emission is practically undetectable from the western footpoint.

Loop thickness. A change in the loop thickness increases the opacity proportionately, which raises the optically thin fluxes without changing the optically thick fluxes greatly. The thickness cannot be increased greatly without the whole loop becoming visible at 15 $\mathrm{GHz}$, in contrast to the observations.

Electron number density. In the optically thin limit changing the number density has the same effect as increasing the magnetic field, while it has little effect in the optically thick limit.

Electron energy cutoffs. Imposing an upper limit to the electron energies present in the distribution has a strong suppression effect on radiation at high frequencies which requires very energetic electrons (high Lorentz factors) if the magnetic field is not strong. Thus increasing the upper energy cutoff, e.g. to $400 \mathrm{keV}$, increases the opacity of the loop top at $15 \mathrm{GHz}$ dramatically and allows it to become visible. By contrast, decreasing the upper cutoff to $100 \mathrm{keV}$ suppresses nearly all emission at $15 \mathrm{GHz}$ when the footpoint field strength 
is $800 \mathrm{G}$. Increasing the lower energy cutoff has little effect on the high-frequency emission since the low-energy electrons do not radiate at $15 \mathrm{GHz}$, but it increases the mean energy of the electrons radiating the optically thick emission at $5 \mathrm{GHz}$ and makes it brighter.

Viewing angle. In practice, the viewing angle can be changed if we change the loop location and orientation. In most cases, the changes affect the model microwave morphology dramatically, because the gyrosynchrotron emission mechanism depends strongly on the angle between the magnetic field and the line of sight.

For simplicity, we simulate a change in the angle of tilt of the loop with respect to the local vertical by changing the latitude of the loop: this will have essentially the same

effect as maintaining the latitude but adding a tilt because the gyrosynchrotron calculations depend most strongly on viewing angle; other parameters, such as the polar angle of the loop, are in fact poorly constrained. The model loop is located at latitude $50^{\circ}$ north while the latitude of the flare was $20^{\circ}$ north. We also simulated model loops located at the heliographic coordinates of the flare. In all cases, no matter what the azimuth of the model loop was, the shape of the projection of the loop on the plane of the sky did not look like the $5 \mathrm{GHz}$ VLA flaring loop. This implies that the observed loop was tilted with respect to the local vertical; we estimate that the tilt angle was about $30^{\circ}$.

Our tests showed that the only way to make the $5 \mathrm{GHz} I$ peak more prominent and at the same time move the peaks of the $5 \mathrm{GHz} I$ and $V$ model emission to the location of the VLA peak is to use the input parameters from the best fit to the OVRO fluxes except to increase the footpoint field strength to $1070 \mathrm{G}$. In this case, the east-central part of the loop becomes optically thick and the convolution with the VLA beam gives the desired morphology. However the resulting fluxes are too high (7 SFU and $27 \mathrm{SFU}$ at $15 \mathrm{GHz}$ and $5 \mathrm{GHz}$ respectively). In order to lower these fluxes we may either make the loop thinner and/or decrease the number density or the energy cutoffs of the energetic electrons. These combinations can easily lead to the correct fluxes but at the same time, they destroy the morphology because they make the central part of the loop optically thin at $5 \mathrm{GHz}$.

\subsection{Polarization images}

Based on gyrosynchrotron theory, the intrinsic polarization of the radio emission should be $x$ mode (with respect to the direction of the magnetic field in the source) as long as the optical depth does not exceed about 4; for large optical depths the polarization should be in the sense of the $o$ mode because the source function for the ordinary mode is higher than for 
the extraordinary (physically, it takes higher-energy electrons to make the $o$ mode optically thick since it has weaker intrinsic opacity). The intrinsic polarization should switch from one side of the loop to the other as the component of the magnetic field along the line of sight changes sign. We do not observe any change in polarization across the loop, and the 5 $\mathrm{GHz}$ loop emission has the same sense of polarization as the $15 \mathrm{GHz}$ footpoints.

The lack of a polarization change from one side of the loop to the other is commonly observed and is believed to result from mode coupling at a point in the corona along the line of sight where the direction of the magnetic field reverses (e.g., White, Thejappa, \& Kundu 1992; Alissandrakis, Nindos, \& Kundu 1993). The fact that the optically thick 5 $\mathrm{GHz}$ loop emission and the presumably optically thin $15 \mathrm{GHz}$ footpoint emission have the same sense of polarization, which is $x$ mode from the sources nearest to earth, indicates that the optical depth of the loop emission is not large enough to make it polarized in the sense of the $o$ mode, i.e., less than about 4.

In order to simulate the effect of polarization inversion on the model $V$ contour plots and profiles (see fig 5 and 6 ), before the convolution we changed the sign of the polarization fluxes which correspond to positive angles between the magnetic field and the line of sight (presumably associated with the limbward part of the flaring loop which is affected by the polarization inversion) The model $5 \mathrm{GHz} V$ profile shows three components after the convolution with the VLA beam: the strongest (middle) component showed negative polarization, in agreement with the observations while the other two components showed positive polarization. The positive components are due to the presence of optically thick regions polarized in the sense of the $o$ mode (the grey circles in fig. 4) and the convolution with the VLA beam does not wash them out. Unfortunately, a direct comparison between the $5 \mathrm{GHz}$ model $V$ profile and the VLA $V$ profile is not conclusive because the strong component of the model $V$ profile is displaced with respect to the peak of the VLA profile. Of course, it is possible to change the model input parameters and make the optical depth of the $5 \mathrm{GHz}$ emission low enough so that the polarization should be in the sense of the $x$ mode along the entire loop. However, in such a case neither the fluxes nor the morphology of the $I$ emission agree with the observations. Furthermore the limited dynamic range of the VLA observations should be considered: the dynamic range of the $5 \mathrm{GHz} V$ map (defined here as peak flux divided by three times the rms map noise $\sigma$ ) is only 8 . The ratio between the peak flux of the strong model $V$ component and the east component is 15 and the ratio between the strong model $V$ component and the west component is 7.9 . Thus if such $o$-mode sources were present, their polarization would be below or at the limit of VLA's dynamic range. 


\section{Conclusions}

We presented VLA observations of a simple small flare at $15 \mathrm{GHz}$ and $5 \mathrm{GHz}$. Additional spectral data were provided by the OVRO Solar Array. At $15 \mathrm{GHz}$, the flare emission came from two compact highly polarized sources which traced the footpoints of the flaring loop. The whole loop itself was seen to emit at $5 \mathrm{GHz}$. The maximum of the $5 \mathrm{GHz}$ emission was associated with the loop top. The reason that the two frequencies appeared different was not instrumental because the $15 \mathrm{GHz}$ baselines were short enough to map, at least partially, a feature with the dimensions of the $5 \mathrm{GHz}$ loop (see the discussion in section 3 ). The different morphologies at the two frequencies reflect the properties of gyrosynchrotron emission from mildly relativistic electrons in an inhomogeneous loop. The footpoints are bright at the higher frequency because the magnetic field of the loop is much higher at the footpoints. At the lower frequency almost the whole loop is optically thick and the maximum occurs at the loop top where the magnetic field is weakest and consequently more energetic electrons are required to produce optically thick emission. The polarized emission was affected by propagation effects which inverted the sense of polarization in the limbward part of the flaring loop. The peak of the $5 \mathrm{GHz} V$ map was located at the loop top, approximately at the same location as the $I$ peak.

We tried to reproduce the VLA spatial structure as well as the OVRO radio spectrum using a simple inhomogeneous gyrosynchrotron model. The observations provided important constraints to the model input parameters. The parameters which determine the location of the loop were chosen so that the shape of the model loop resembled the shape of the loop-like $5 \mathrm{GHz}$ VLA source. The distance between the $15 \mathrm{GHz}$ VLA sources gave the footpoint separation of the model loop. The spectral index of the OVRO total power data provided the power law energy spectral index of the model nonthermal electrons. The other input parameters were free. The best fit to the spectral data as well as a qualitatively satisfactory agreement with the morphology of the VLA maps was achieved using the following values: the photospheric magnetic field strenth was $870 \mathrm{G}$. The heights above the solar surface of the two lines of force which formed the loop were $1.9 \times 10^{9} \mathrm{~cm}$ and $1.8 \times 10^{9}$ $\mathrm{cm}$. The transverse dimension of the loop was $5.25 \times 10^{7} \mathrm{~cm}$. The density of the energetic electrons was $7.9 \times 10^{7} \mathrm{~cm}^{-3}$ and their low and high energy cutoffs were $8 \mathrm{keV}$ and $210 \mathrm{keV}$ respectively. The agreement between the computed and observed fluxes was very good at high frequencies. At low frequencies where the exact geometric shape of the loop is more important, the discrepancy between the computations and the observations was as high as a factor of 2.5 probably due to our simplified loop model.

This model reproduced in a satisfactory way most of the features of the VLA observations. At $15 \mathrm{GHz}$, the model emission showed optically thin highly-polarized 
emission from the footpoints of the loop. At $5 \mathrm{GHz}$ the model emission came from the entire loop. This picture has been observed repeatedly. For example Shevgaonkar \& Kundu (1985) observed a burst with the VLA which showed two compact optically thin sources at $15 \mathrm{GHz}$ located near the edges of a larger presumably optically thick source observed at $5 \mathrm{GHz}$. Single frequency VLA observations at $15 \mathrm{GHz}$ (e.g. Marsh, \& Hurford 1980) have shown compact sources located between the $\mathrm{H} \alpha$ kernels of the flare. Generally, loop top emission is favored when the magnetic field from top to footpoints of the loop does not increase rapidly and the accelerated particles have a nearly isotropic pitch angle (Petrosian 1982). The emission continues to originate from the loop top until a large number of trapped electrons is scattered into the loss cone by additional turbulence or Coulomb deflections (Holman, Kundu, \& Papadopoulos 1982).

The model field strenth was $870 \mathrm{G}$ at the photosphere and $282 \mathrm{G}$ at the loop top. Thus, it was strong enough so that the $5 \mathrm{GHz}$ emission came from low harmonics of the gyrofrequency (harmonics $3-7$ ). The harmonic structure is especially prominent in the $I$ model (see fig. 4, top panel). This harmonic structure is much less evident when the model is convolved with the VLA beam, and then the $I$ and $V$ peak fluxes lie close to the loop top in rough agreement with the observations (the VLA $I$ and $V$ peaks were somewhat displaced with respect to the model peaks, however). The traditional interpretation of microwave bursts assumes that gyrosynchrotron emission comes from high harmonics (10-100) of the gyrofrequency (e.g., see Krüger 1979; Dulk 1985). Our modeling clearly shows that this is not always the case. The fact that the model photospheric field strenth is close to the KPNO magnetogram values of the magnetic field associated with the $15 \mathrm{GHz}$ VLA sources (800-950 G) makes us confident that indeed most of the $5 \mathrm{GHz}$ emission comes from low harmonics of the gyrofrequency.

One might think that this result is not consistent with the technique we used to derive the energy spectral index of the energetic electrons: the expressions by Dulk (1985) are only valid for harmonics greater than about 10 . We computed model flux spectra with $\delta=3$, 3.5, 3.9 and 4.4. For the $\delta<3.7$ models we tried to fit the OVRO high-frequency fluxes by decreasing one or more of the following input parameters: loop thickness, magnetic field strength, electron density and the energy cutoffs. For the $\delta>3.7$ models we increased these parameters. We tried all possible combinations, but using the chi-square criterion, we found that the model with $\delta=3.7$ that we presented in section 4 was the best fit case. This result suggests that the error in the calculation of $\delta$ using the Dulk (1985) formula is smaller than other errors which may come from inhomogeneities that were not taken into account and the simplified loop geometry.

The high magnetic fields also explain the interesting feature that the $5 \mathrm{GHz}$ emission 
did not extend all the way down to the feet of the loop. Figs 4 and 5 provide the explanation. At the eastern edge of the loop we have gyrosynchrotron emission from the third harmonic of the gyrofrequency. At the very edge of the loop the angle between the magnetic field and the line of sight $\theta$ is not big enough to give high $5 \mathrm{GHz}$ emission. But only $6 \times 10^{7} \mathrm{~cm}$ higher, the optical depth becomes almost three times larger resulting in a higher $5 \mathrm{GHz}$ flux. The same effect is repeated for about $10^{9} \mathrm{~cm}$ along the loop: harmonics $3-5$ produce local $I$ peaks when the angle $\theta$ is sufficient. The convolution of the model $I$ profile with the VLA beam washes out those local peaks and makes the bright $5 \mathrm{GHz}$ emission appear not to overlap with the peak $15 \mathrm{GHz}$ emission.

Magnetic trapping may also contribute to restricting the size of the $5 \mathrm{GHz}$ source. The magnetic field strength of our model is $B_{1}=870 \mathrm{G}$ at the footpoints of the loop and $B_{0}=280 \mathrm{G}$ at the loop top thus the mirror ratio is about 3 . Consequently, the loss cone angle is $\alpha_{0}=\arcsin \left(B_{0} / B_{1}\right)^{1 / 2} \approx 35^{\circ}$. Electrons with pitch angles higher than $35^{\circ}$ are trapped in the magnetic loop and mirror back and forth between the same magnetic field strength levels at both ends of the flux tube. If the loop is asymmetric, this effect prevents trapping of electrons at the part of the loop which is confined by the footpoint with the strongest field and the height where the field is equal to the field strength of the other footpoint. In this case, the $15 \mathrm{GHz}$ emission comes from a subset of electrons which are in the loss cone and therefore can reach the footpoint area. Independent evidence for this scenario has been provided by Lee, Gary, \& Shibasaki (1999) who studied two other flares observed with OVRO.

Our modeling also provides important constraints on the magnetic field geometry. The fact that we obtained a match to the observed morphology with our model loop at N50E50 while the real location of the flare was N20E50 suggests that the loop was tilted about $30^{\circ}$ with respect to the local vertical. This indirect result appears more reliable than the $65^{\circ}$ tilt that we found using the formulas by Loughhead, Wang, \& Blows (1983) - It is also interesting that both the thickness of the loop and its transverse dimension are small compared to the footpoint separation. We ended up with such small values because our scope was to reproduce the VLA morphology as well as the fluxes. Thicker loops can also give satisfactory morphologies at 5 and $15 \mathrm{GHz}$ (provided that we change the other free parameters appropriately) but the resulting fluxes are not consistent with the OVRO values. Of course these small dimensions are below the VLA spatial resolution. The same is also true of the fine structure of the $5 \mathrm{GHz}$ model emission which was washed out after the convolution with the VLA beam.

We note that the density of energetic electrons of our model was relatively high and only 25-40 times lower than usual electron densities of the ambient thermal plasma in 
coronal loops (e.g. see, Kano, \& Tsuneta, 1995, 1996; Aschwanden et al. 1999). However, Aschwanden \& Benz (1997) found that the thermal electron density in 44 soft X-ray flaring loops was $0.2-2.5 \times 10^{11} \mathrm{~cm}^{-3}$, i.e. a factor of about 1700 higher than the density of nonthermal electrons of our model. This high density was necessary to make the $5 \mathrm{GHz}$ emission optically thick because the loop needed to be thin in order not to have very large fluxes at $5 \mathrm{GHz}$. The resulting loop is very thin $\left(\sim 1.4^{\prime \prime}\right)$ compared to its height $\left(\sim 25^{\prime \prime}\right)$; however, a thicker loop does not produce a match to the VLA observations at both 5 and $15 \mathrm{GHz}$, or to the spectrum observed by OVRO. A loop as thin as the one of the best fit model is needed in order to reproduce the entire observational dataset. The relatively high density suggests that both the acceleration process and trapping of energetic electrons were efficient in this flaring loop. Furthermore, the absence of electrons above $210 \mathrm{keV}$ is necessary to explain why no emission was observed from loop top at $15 \mathrm{GHz}$.

The good agreement between our simple gyrosynchrotron computations and the observations verifies that the gyrosynchrotron mechanism combined with the magnetic field geometry is, by far, the most important factor which determines the morphology of microwave bursts. However, our model computations have not exhausted the subject. For example, if suitable magnetic field data existed, it would be interesting to constrain the model loop geometry with nonlinear force-free extrapolations of the photospheric field. A still better agreement with the observations is possible, if the effects of the ambient medium are taken into account. For this purpose soft X-ray observations would be valuable. Furthermore, models which incorporate the time evolution of the emission will help us to confront the problems of energy release and particle acceleration in solar flares.

\section{ACKNOWLEDGMENTS}

This research at the University of Maryland was carried out with support from NSF grant ATM 96-12738 and NASA grants NAG 5-7370, NAG 5-6257 and NAG 5-7901. The OVRO observations were made possible through support from NSF grants ATM 97-96213 and AST 97-96238, and NASA grant NAG5-6381 to New Jersey Institute of Technology. SMW acknowledges gratefully the assistance in setting up the high-time-resolution observations provided by Tim Bastian and the VLA operations staff. 


\section{REFERENCES}

Alissandrakis, C. E. 1986, Sol. Phys., 104, 207.

Alissandrakis, C. E., \& Preka-Papadema, P. 1984, A\&A, 139, 507.

Alissandrakis, C. E., Nindos, A., \& Kundu, M. R. 1993, Sol. Phys., 147, 343.

Aschwanden, M. J., \& Benz, A. O. 1997, ApJ, 480, 825.

Aschwanden, M. J., Newmark, J. S., Delaboudiniere, J.-P., Neupert, W. M., Klimchuk, J. A., Gary, G. A., Portier-Fozzani, F., \& Zucker, A. 1999, ApJ, 515, 842.

Bastian, T. S., \& Kiplinger, A. 1991, in R. Winglee, A. Kiplinger, (eds) "Max 1991 Workshop No 3", Estes Park, CO., 153.

Bastian, T. S., Benz, A. O., \& Gary, D. E. 1998, ARA\&A, 36, 131.

Belkora, L. 1997. ApJ, 481, 532.

Dulk, G. A. 1985, ARA\&A, 23, 169.

Franciosini, E., \& Chiuderi-Drago, F. 1995, A\&A, 297, 535.

Hanaoka, Y. 1997, Sol. Phys., 173, 319.

Holman, G. D., Kundu, M. R., \& Papadopoulos, K. 1982, ApJ, 257, 354.

Jones, K. L., Stewart, R. T., Nelson, G. J., \& Duncan, R. A. 1994, MNRAS, 269, 1145.

Kano, R., \& Tsuneta, S. 1995, ApJ, 454, 934.

Kano, R., \& Tsuneta, S. 1996, PASJ, 48, 535.

Klein, K.-L. 1987, A\&A, 183, 341.

Kundu, M. R., \& Vlahos, L. 1982, SSRv, 32, 405.

Kundu, M. R., Schmahl, E. J., Velusamy, T., \& Vlahos, L. 1982, A\&A, 108, 188.

Krüger, A. 1979. "Introduction to Solar Radio Astronomy and Radio Physics", Reidel.

Lee, J., Gary, D. E., \& Shibasaki, K. 1999, ApJ, submitted.

Loughhead, R. E., Wang, J. L., \& Blows, G. 1983, ApJ, 274, 883.

Marsh, K. A., \& Hurford, G. J. 1980, ApJ, 240, L111. 
Nishio, M., Yaji, K., Kosugi, T., Nakajima, H., \& Sakurai, T. 1997, ApJ, 489, 976.

Nitta, N., White, S. M., Schmahl, E. J., \& Kundu, M. R. 1991, Sol. Phys., 132, 125.

Petrosian, V. 1982, ApJ, 255, L85.

Preka-Papadema, P., \& Alissandrakis, C. E. 1988, A\&A, 191, 365.

Preka-Papadema, P., \& Alissandrakis, C. E. 1992, A\&A, 257, 307.

Preka-Papadema, P., Alissandrakis, C. E., Dennis, B. R., \& Kundu, M. R. 1997, Sol. Phys., $172,233$.

Razin, V. A. 1960, Izv. Vys. Ucheb. Zav. Radiofiz., 3, 584.

Schmahl, E. J., Kundu, M. R., \& Dennis, B. R. 1986, Adv. Space Res., 6, 143.

Shevgaonkar, R. K., \& Kundu, M. R. 1985, ApJ, 292, 733.

Stähli, M., Gary, D. E., \& Hurford, G. J. 1989, Sol. Phys., 120, 351.

Takakura, T., Nishio, M., Nakajima, H., Enome, S., \& Shibasaki, K. 1994, PASJ, 46, 653.

Velusamy, T., Kundu, M. R. 1982, ApJ, 258, 388.

Wang, H., Gary, D. E., Lim, J., \& Schwartz, R. A. 1994, ApJ, 433, 379.

Wang, H., Gary, D. E., Zirin, H., Kosugi, T., Schwartz, R. A., \& Linford, G. 1995, ApJ, 444, L115.

White, S. M., Thejappa, G., \& Kundu, M. R. 1992, Sol. Phys., 138, 163.

Zheleznyakov, V. V. 1970, "Radio Emission of the Sun and Planets", Pergamon Press, Oxford. 


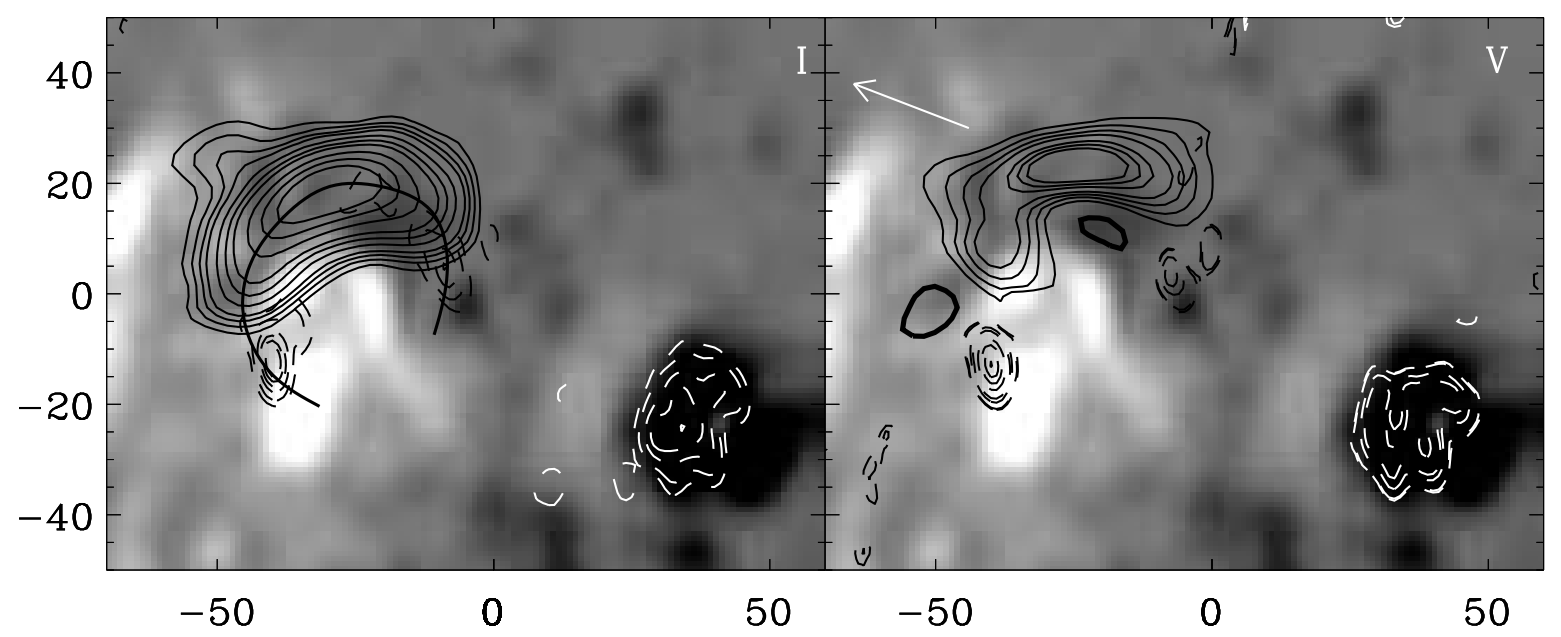

Fig. 1.- Contour plots of the VLA flare at the time of maximum overlaid on a Kitt Peak photospheric magnetogram. The magnetogram was observed about 5 min after the end of the flare. The total intensity $I$ snapshot maps are on the left and the circular polarization $V$ maps on the right. The solid contours show the $5 \mathrm{GHz}$ emission and the dashed contours show the $15 \mathrm{GHz}$ emission. In the $5 \mathrm{GHz} V$ map the thick contours represent positive brightness temperatures. The white contours in both images show the sunspot-associated emission at $15 \mathrm{GHz}$. The contour levels in the $I$ maps are $(2.25,3,3.75,4.5,6,7.5,9,12,15$, $24) \times 10^{6} \mathrm{~K}$ and $(0.2,0.4,0.9,1.4,1.9,2.3) \times 10^{6} \mathrm{~K}$ for $5 \mathrm{GHz}$ and $15 \mathrm{GHz}$ respectively. The contour levels in the $V$ maps are $(-0.55,-0.45,-0.40,-0.25,-0.20,-0.15,-0.07,0.07) \times 10^{5} \mathrm{~K}$ and $(-23,-18,-14,-9,-4.5,-3.3,-1.6,-1.2) \times 10^{5} \mathrm{~K}$ for $5 \mathrm{GHz}$ and $15 \mathrm{GHz}$ respectively. The axes labels denote seconds of arc from the VLA phase-tracking center. North is up, West to the right. The white arrow indicates the direction of the limb. The VLA profiles of fig. 4 have been computed along the solid curve which is present on the $I$ maps of this figure. 


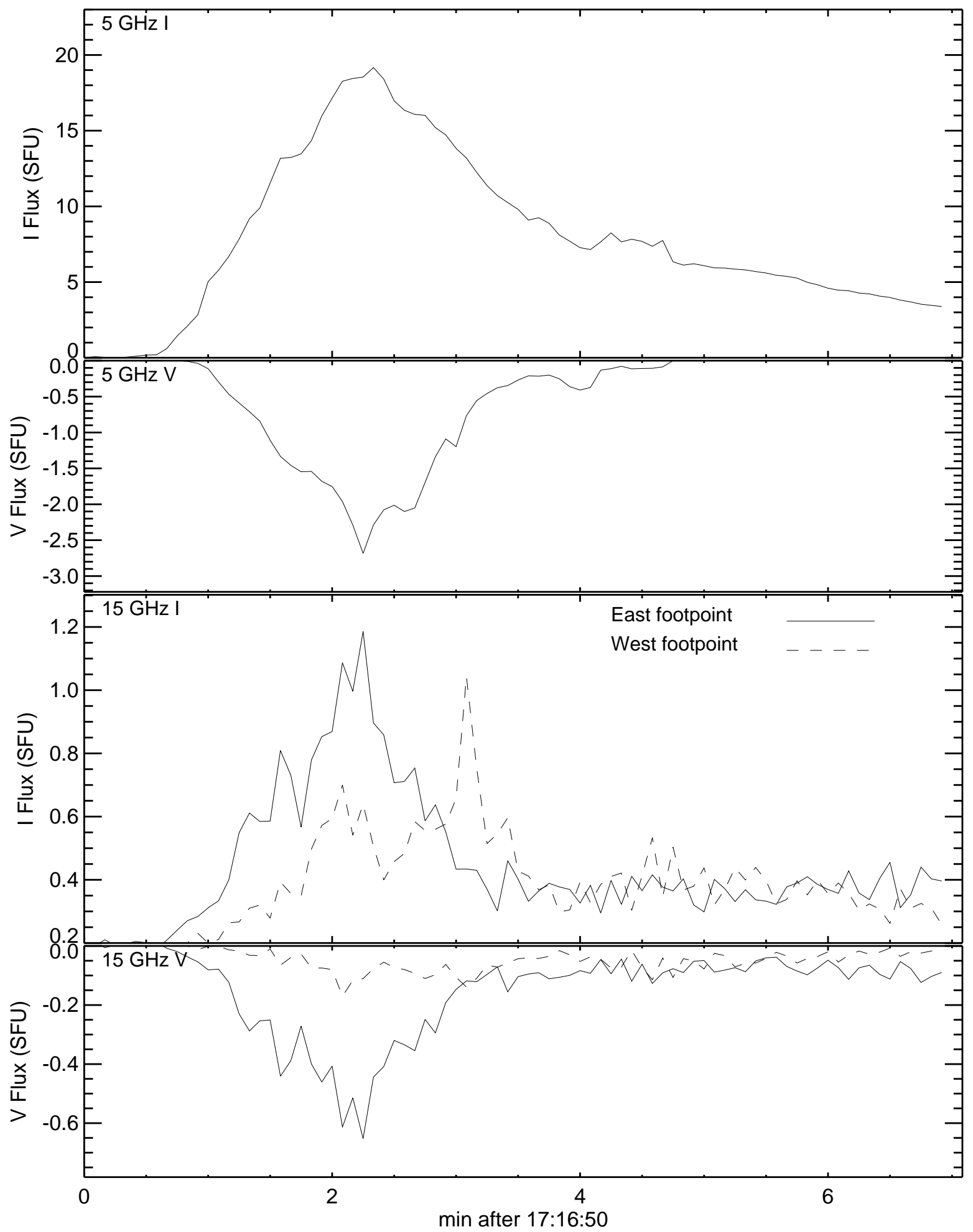

Fig. 2.- Time profiles of the VLA flare. From top to bottom: $5 \mathrm{GHz} I, 5 \mathrm{GHz} V, 15$ $\mathrm{GHz} I$ and $15 \mathrm{GHz} V$ time profiles. In the $15 \mathrm{GHz}$ profiles the solid lines show the time evolution of the eastern footpoint emission and the dashed lines the time evolution of the western footpoint emission. 


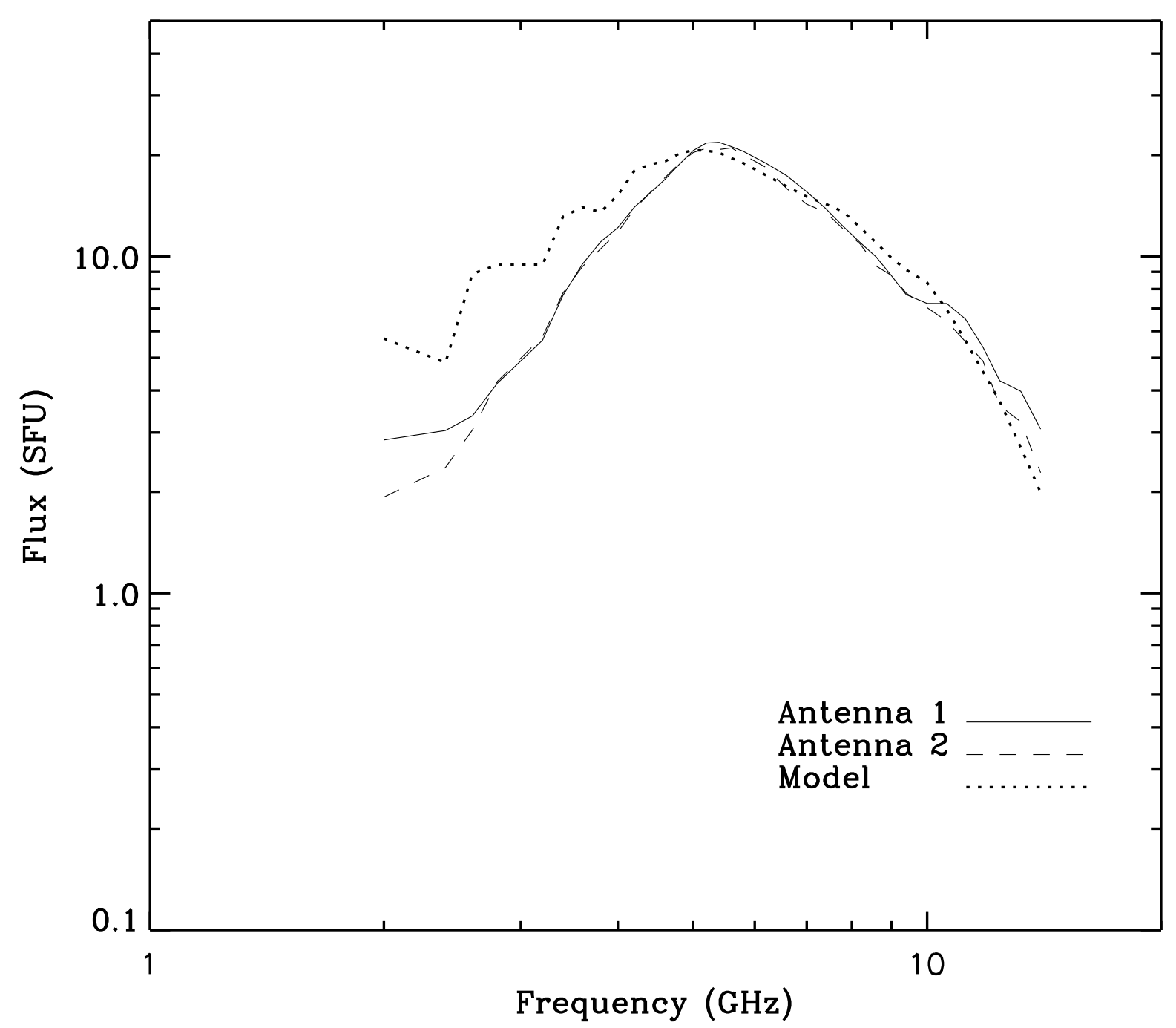

Fig. 3.- OVRO total power spectra from antennas 1 and 2, at the time of maximum. The dotted curve is the best fit model to the OVRO spectra. The model was a magnetic loop located at heliographic longitude $50^{\circ}$ east and latitude $50^{\circ}$ north and with a $45^{\circ}$ orientation (azimuth) with respect to the local north. The photospheric footpoint magnetic field strength was $870 \mathrm{G}$. The heights above the solar surface of the two lines of force which formed the loop were $1.9 \times 10^{9} \mathrm{~cm}$ and $1.8 \times 10^{9} \mathrm{~cm}$. The distance between the footpoints was $2.64 \times 10^{9}$ $\mathrm{cm}$ and the transverse dimension of the loop was $5.25 \times 10^{7} \mathrm{~cm}$. The energy spectral index of the energetic electrons was 3.7 and their density was $7.9 \times 10^{7} \mathrm{~cm}^{-3}$. The low and high energy cutoffs of the nonthermal electrons were $8 \mathrm{keV}$ and $210 \mathrm{keV}$ respectively. 


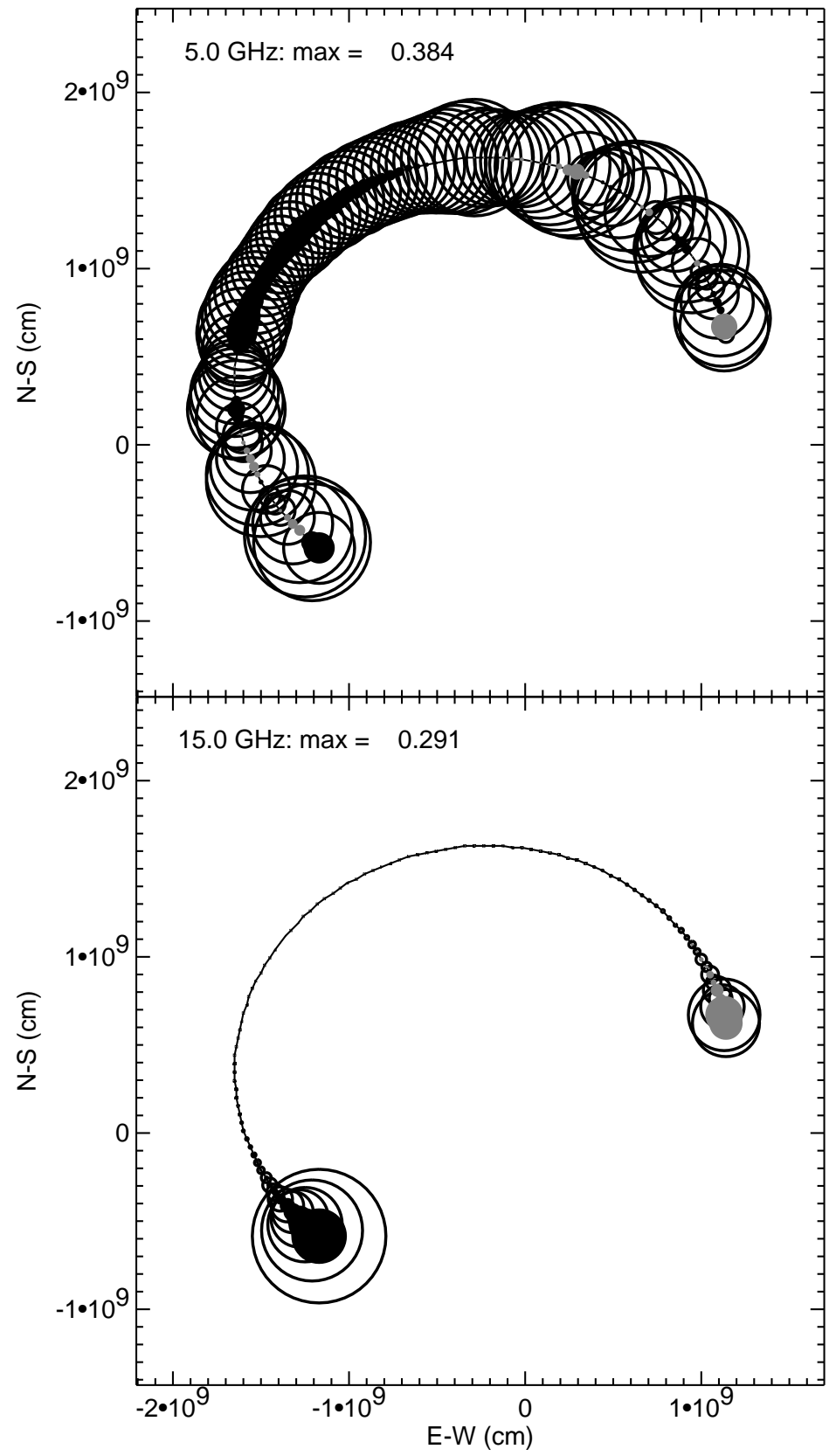

Fig. 4.- The morphology at $5 \mathrm{GHz}$ and $15 \mathrm{GHz}$ of the model fit to the spectrum. The loop total intensity and circular polarization fluxes have been plotted as circles on the plane of the sky. The open circles represent total intensity flux and the filled circles represent circular polarization flux (the black circles indicate $x$-mode polarization and the grey circles indicate $o$-mode polarization). At each frequency, the radius of each circle is proportional to the corresponding model $I$ or $V$ flux. 


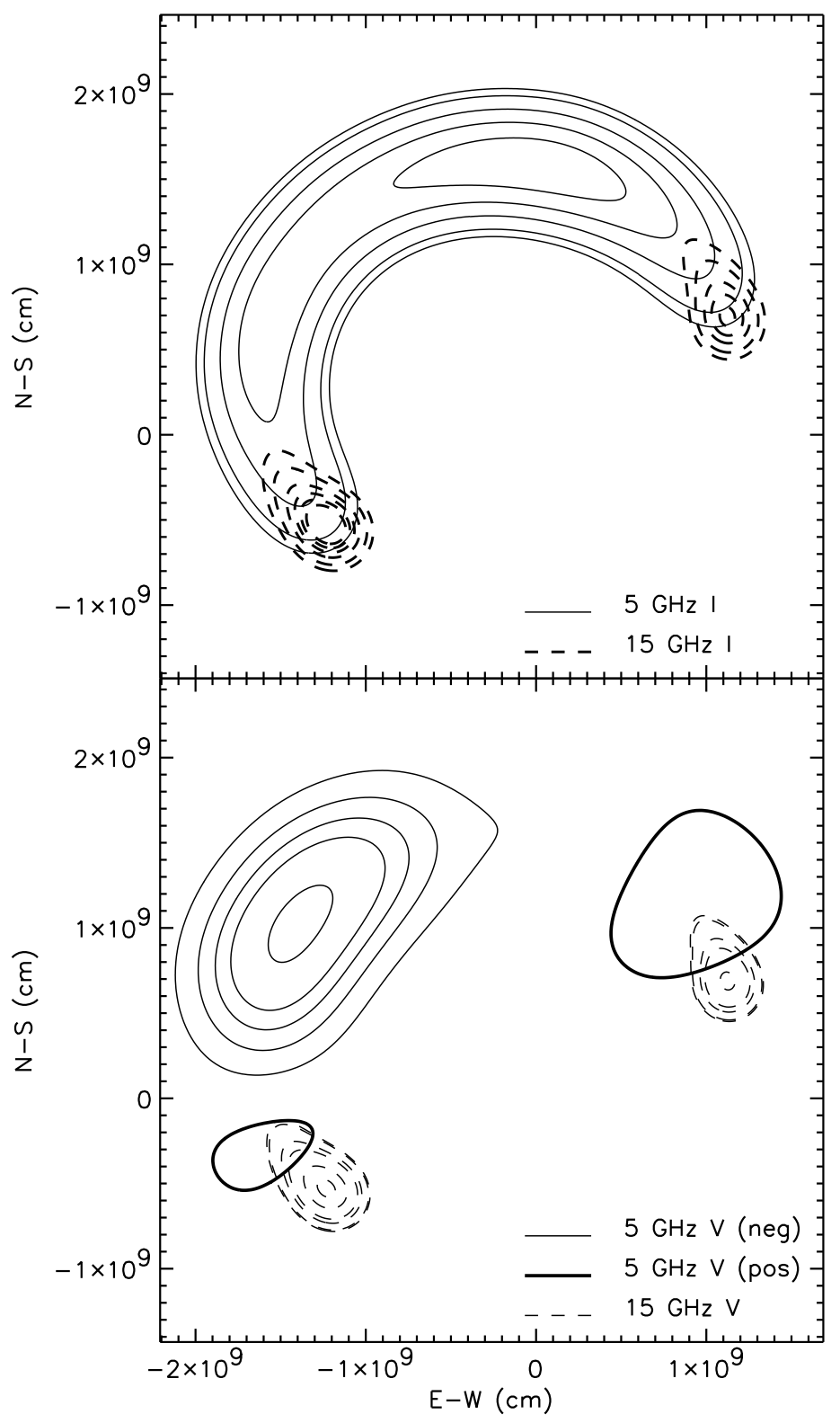

Fig. 5.- Contour plots of the model morphologies presented in fig. 4. For comparison with the observations, each model has been convolved with the appropriate VLA beam. In order to simulate the effect of polarization inversion on the model $V$ maps, before the convolution we changed the sign of the polarization fluxes which correspond to positive angles between the magnetic field and the line of sight (presumably associated with the limbward part of the flaring loop which is affected by the polarization inversion). The contour levels in the $I$ maps are $(1.28,1.92,2.56,3.2,3.84) \mathrm{SFU} /$ beam and $(0.05,0.1,0.2,0.3,0.4,0.5) \mathrm{SFU} / \mathrm{beam}$ for $5 \mathrm{GHz}$ and $15 \mathrm{GHz}$ respectively. The contour levels in the $V$ maps are $(-0.15,-0.3,-0.45$, $-0.6,-0.9,0.02) \mathrm{SFU} /$ beam and $(-0.3,-0.2,-0.1,-0.07,-0.035,-0.03) \mathrm{SFU} /$ beam for $5 \mathrm{GHz}$ and $15 \mathrm{GHz}$ respectively. 


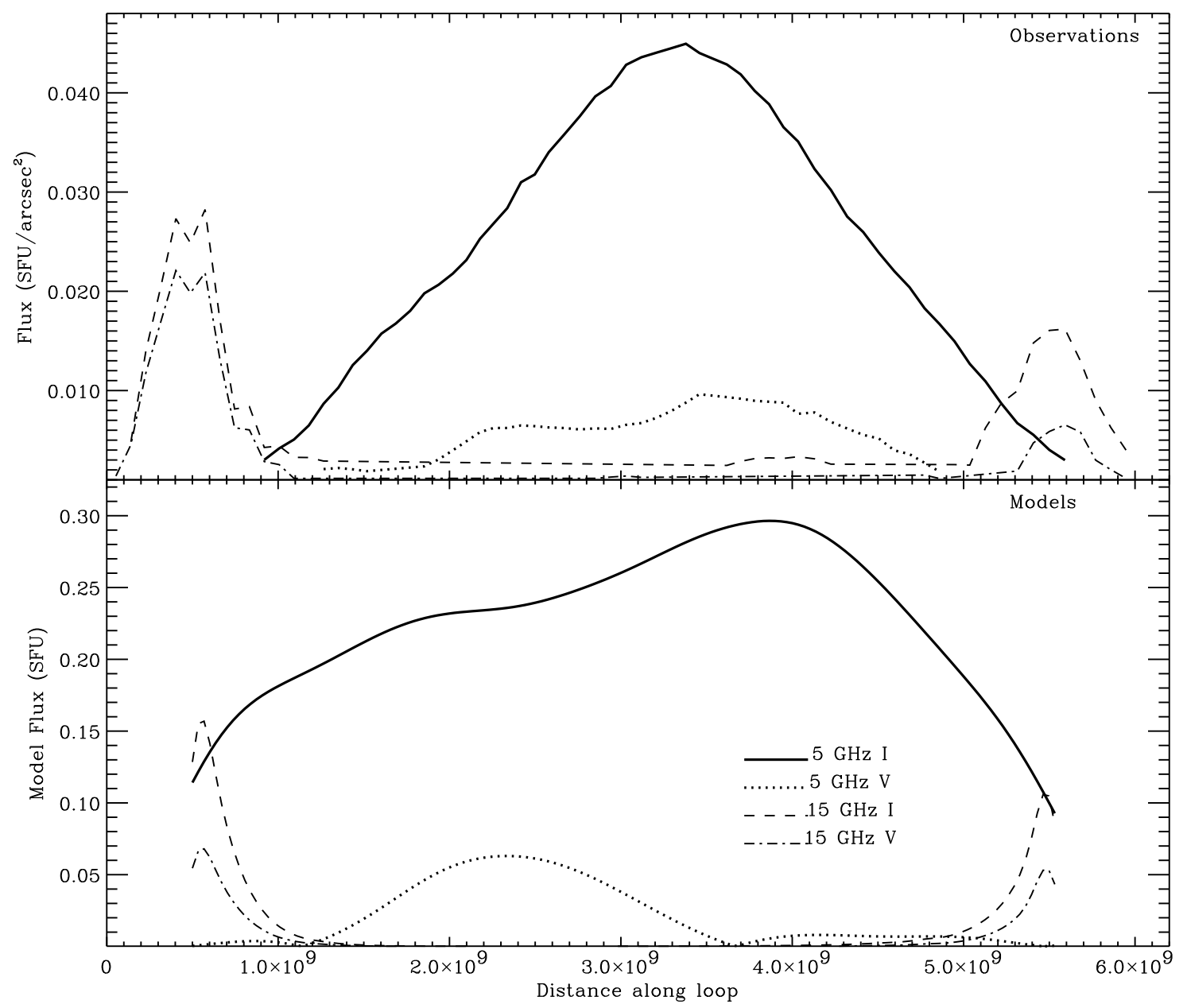

Fig. 6.- Top panel: One-dimensional representation of the VLA flare at the time of maximum. These profiles were computed along the solid curve shown in the left frame of fig. 1. Bottom panel: spatial profiles of the models of fig. 4 as a function of the distance along the loop. For comparison with the observations, each profile has been convolved with the appropriate VLA beam. The inversion of circular polarization was treated as in fig. 5. In both panels, we present the absolute values of the $V$ profiles. For a better comparison, we have divided the VLA values with the appropriate beam. 